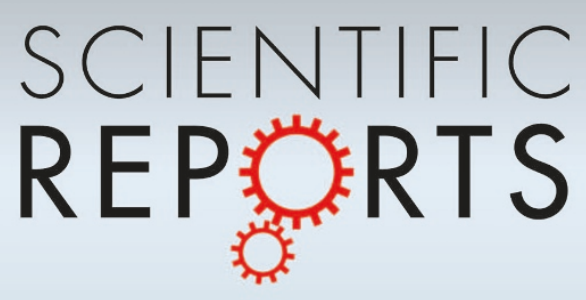

\title{
OPEN Majorana modes in a topological \\ TOPOLOGICAL \\ INSULATORS insulator/s-wave superconductor heterostructure
}

SUBJECT AREAS:

SUPERCONDUCTING PROPERTIES AND MATERIALS

Received

11 June 2014

\author{
Zheng-Zao Li1,2, Fu-Chun Zhang ${ }^{2,3} \&$ Qiang-Hua Wang ${ }^{1,2}$
}

Accepted

11 August 2014

Published

15 September 2014

Correspondence and requests for materials should be addressed to

Q.H.W. (qhwang@ nju.edu.cn)

\begin{abstract}
${ }^{1}$ National Laboratory of Solid State Microstructures, Nanjing University, Nanjing, 210093, China, ${ }^{2}$ Collaborative Innovation Center of Advanced Microstructures, Nanjing 210093, China, ${ }^{3}$ Department of Physics, Zhejiang University, Hangzhou, China.
\end{abstract}

In a recent experiment, signatures of Majorana fermion (MF) were found in the vortex core threading a heterostructure composed of $\boldsymbol{n}$ layers of topological insulator (TI) deposited on a bulk s-wave superconductor. Here we provide strong theoretical support to the experiment. First, we demonstrate that MF modes appear on both top and bottom layers of TI, and are well separated for $n \sim 6$. The top MF becomes more extended with increasing $n$, in agrement with the experiment. Second, we show both analytically and numerically that right at the vortex core the MF mode is always accompanied by another low energy bound state, leading to a zero-bias peak plus a side peak in the local density of states (LDOS) therein. However, a local scalar impurity at the core can wipe out the accompanying side-peak state while leaving the zero-energy MF mode intact. Consequently the LDOS becomes symmetric about the fermi level, and the peak does not branch near the vortex core, in agreement with the experiment. Finally but unfortunately, while the MF is extremely stable against a single local impurity, the stability in terms of the critical impurity strength is reduced drastically for a moderate concentration (e.g., 10\%) of impurities.

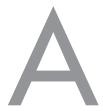

Majorana fermion (MF) is an anti-particle of itself, originally proposed as a candidate for neutrino ${ }^{1}$. Recently it was proposed that MF may be generated in some particular condensed matter systems. For example, a zero energy MF mode can appear in the vortex core of the s-wave superconductor/bulk topological insulator heterostructure ${ }^{2}$. As a variant, MF may also appear at the open ends of a semiconducting nano-ribbon with strong spin-orbital coupling, in proximity to an s-wave superconductor and under a high magnetic field ${ }^{3}$. Apart from such hetero-structures, MF may appear in vortices of pure systems, such as the superfluid ${ }^{3} \mathrm{He}-\mathrm{B}^{4}$ and the potential $p_{x}+i p_{y}$-wave superconductor $\mathrm{Sr}_{2} \mathrm{RuO}_{4}{ }^{5}$. The renewed extensive interests in search of MFs are mainly driven by the potential application of MFs. Since the MFs obey non-Abelian statistics, they can be utilized to perform fault-tolerant topological quantum computing ${ }^{6}$. In addition, the MF mode also leads a plethora of new intriguing effects, such as fractional Josephson effect ${ }^{7,8}$, resonant Andreev reflection ${ }^{9,10}$, and resonant crossed Andreev reflection ${ }^{11,12}$.

Recently important experimental progresses have been made for the topological insulator/s-wave superconductor heterostructure ${ }^{13,14}$. In the earlier theoretical proposal ${ }^{2}$, a thin layer of superconductor (SC) is to be deposited on a bulk topological insulator (TI). However, experimentally it is difficult to prepare a pristine bulk TI (such as $\mathrm{Bi}_{2} \mathrm{Se}_{3}$ ), while it is much easier to prepare a bulk SC (such as $\mathrm{Ne}_{2} \mathrm{Se}_{3}$ ). Therefore, in Ref. 13 a few (quintuple) layers of TI was deposited on the bulk SC substrate, resulting in a TI/SC configuration. Signatures of both superconductivity and the surface states of TI (away from the Fermi level) were observed ${ }^{13}$. More recently, the structure is further improved by replacing $\mathrm{Bi}_{2} \mathrm{Se}_{3}$ with $\mathrm{Bi}_{2} \mathrm{Te}_{3}{ }^{14}$. Scanning tunneling microscopy measurements (STM) of the local density of states (LDOS) on the top layer revealed that the zero-bias peak in the LDOS is perfectly symmetric and does not branch in energy up to a finite distance away from the vortex core, if the number of TI layers $n \geq 4$. This non-branching behavior is in contrast to the case of $n<4$, in which the branching begins right away from the vortex core as in usual $s$-wave superconductors. The non-branching behavior was argued as the signature of MF. The experiment appears very promising. However, in order for the heterostructure to be applied, a few important issues need to be addressed. First, since the zero-bias-peak appears for any case of $n$ in the experiment, it is necessary to identify/falsify the zero energy bound state as a MF, in addition to the identification based on the non-branching behavior. Second, it is necessary to determine and understand the optimal number of TI layers to realize MF. Finally, in a more general respect, there are inevitably imperfections or impurities in real samples. For applications it is important to understand how impurities would destroy a MF mode. We should 

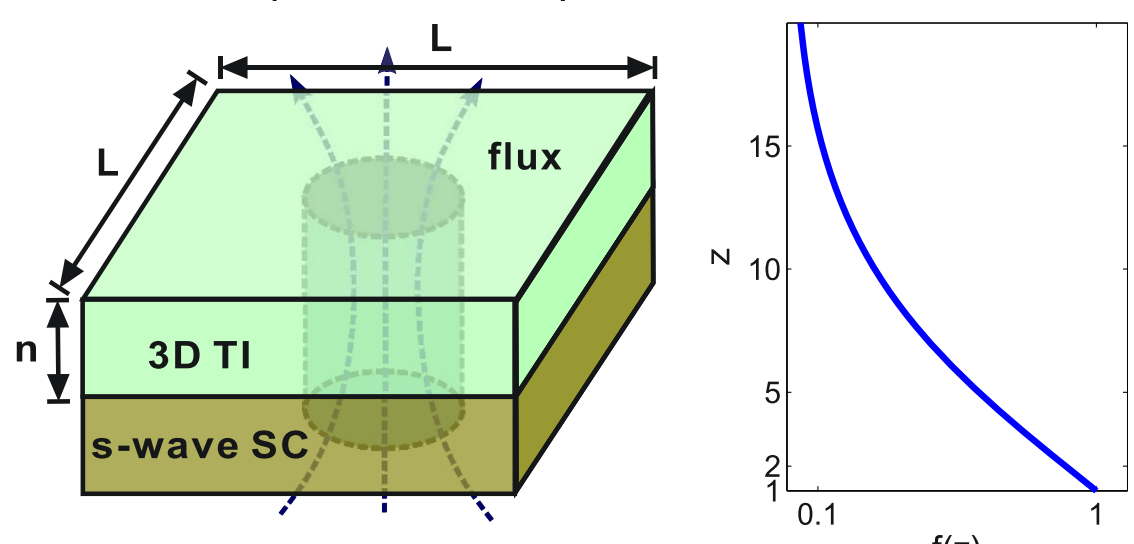

$f(z)$

Figure $1 \mid$ (a) Schematic plot of a vortex threading the $L \times L \times n$ TI above the SC substrate (gray box). (b) The assumed proximity function $f(z)$.

mention that according to the experiment, the Fermi level is away from the Dirac point of the TI surface states (in fact close but below the conduction band). The situation is very different to the SC/TI/SC configuration with the Fermi level right at the Dirac point addressed elsewhere ${ }^{15-17}$.

Here we address the above issues theoretically. First, we demonstrate that MF modes appear on both top and bottom layers of TI, and are well separated for a moderate $n$. The top MF becomes more extended with increasing $n$, in agrement with the experiment. Second, we show both analytically and numerically that right at the vortex core the MF mode is always accompanied by another low energy bound state, leading to a zero-bias peak plus a side peak in the local density of states (LDOS) therein. However, a local scalar impurity at the core can wipe out the accompanying side-peak state while leaving the zero-energy MF mode intact. Consequently the LDOS becomes symmetric at the vortex core, and the peak does not branch near the vortex core, in agreement with the experiment. Finally but unfortunately, while the MF is extremely stable against a single local impurity, the stability in terms of the critical impurity strength is reduced drastically for a moderate concentration of impurities.

The 3d heterostructure. The in-plane structure of the experimental TI is a triangle lattice, and each unit cell contains a quintuple layer in the out-of-plane direction. For convenience but without loss of generality, we model the $3 \mathrm{D}$ TI by a simple cubic lattice, with the hamiltonian

$$
\begin{aligned}
H_{0}= & \frac{1}{2} \sum_{\mathbf{r}, \mathbf{b}}\left[\psi_{\mathbf{r}}^{\dagger} t_{\mathbf{b}}\left(\Gamma_{0}-i \Gamma_{\mathbf{b}}\right) \psi_{\mathbf{r}+\mathbf{b}}+\text { h.c. }\right] \\
& +\sum_{\mathbf{r}} \psi_{\mathbf{r}}^{\dagger}\left[\left(m-\sum_{\mathbf{b}} t_{\mathbf{b}}\right) \Gamma_{0}-\mu\right] \psi_{\mathbf{r}} .
\end{aligned}
$$

Here $\psi_{\mathbf{r}}$ is a four-spinor annihilation field at site $\mathbf{r}=(x, y, z), \mathbf{b}=\hat{x}, \hat{y}, \hat{z}$ is a bond vector connecting nearest-neighbor sites, and $t_{\mathrm{b}}$ is the hopping parameter along bond $\mathbf{b}$. On the other hand, $\Gamma_{0}=\eta_{3} \otimes \sigma_{0}$ and $\Gamma_{\mathbf{b}}=\eta_{1} \otimes \sigma_{\mathbf{b}}$ are four Dirac matrices. The Pauli matrices $\eta(\sigma)$ act on orbital (spin) basis. The parameters $m$ controls the topology of the insulator, and finally $\mu$ is the chemical potential. We take $\hat{z}$ as the direction normal to the quintuple layer in $\mathrm{Bi}_{2} \mathrm{Se}_{3}$ or $\mathrm{Bi}_{2} \mathrm{Te}_{3}$, and we model the anisotropy by taking $\left(t_{\hat{x}}, t_{\hat{y}}, t_{\hat{z}}\right)=\left(t_{\|}, t_{\|}, t_{\perp}\right)$.

We assume that the SC substrate contacting the bottom layer leads to an effective $s$-wave pairing hamiltonian

$$
H_{p}=\sum_{\mathbf{r}} \psi_{\mathbf{r}}^{\dagger} \Delta(\mathbf{r}) i \sigma_{\hat{y}} \psi_{\mathbf{r}}^{\dagger}+\text { h.c. }
$$

Under an applied magnetic field along $\hat{z}$, vortices appear and thread the heterostructure. Experimentally the vortices are dilute for a mag- netic field of the order of Tesla. Thus we consider an isolated vortex line in an $L \times L \times n$ cubic lattice, as schematically shown in Fig. 1(a). The pairing potential is written as,

$$
\Delta(\mathbf{r})=\Delta_{0} \tanh \left(r_{\|} / \xi\right) e^{i \phi_{\mathbf{r}_{\|}}} f(z),
$$

where $\Delta_{0}$ is the pairing amplitude in $\mathrm{SC}, \mathbf{r}_{\|}=(x, y)$ is the planar position vector (the origin of which is set at the center of the $L \times L$ lattice) with azimuthal angle $\phi_{\mathbf{r}_{\|}}, \xi$ is the coherence length of the SC substrate, and finally $f(z)$ is a proximity function. The concrete form of $f(z)$ is unknown. However it is reasonable to take $f(n)=\Delta_{n} / \Delta_{0}$, where $\Delta_{n}$ is the pairing gap measured at the top layer of a heterostructure with $n$ layers of TI. In qualitative accordance to the experiment ${ }^{13,14}$, we take such a function as plot in Fig. 1(b). We assume $\xi=$ $\xi_{0} / f(z)$, where $\xi_{0}$ is the coherence length in the SC substrate, to reflect the fact that the effective pairing gap varies along $z$. In the London limit the magnetic field leads to a vector potential $\mathbf{a}(\mathbf{r})=\pi(-y, x, 0) /$ $\left(2 L^{2}\right)$ in dimensionless units. This amounts to a unit of flux $\phi_{0}=h c /$ $2 e$ threading the planar area. The vector potential couples to electrons minimally via

$$
t_{\mathbf{b}} \rightarrow t_{\mathbf{b}} e^{i \mathbf{b} \cdot \mathbf{a}(\mathbf{r}+\mathbf{b} / 2)}
$$

in the first line of Eq.(1).

The complete hamiltonian is $H=H_{0}+H_{p}$. Formally we rewrite it in the Nambu space as

$$
H=\sum_{\mathbf{r}} \Psi_{\mathbf{r}}^{\dagger} h\left(\mathbf{r}, \mathbf{r}^{\prime}\right) \Psi_{\mathbf{r}^{\prime}}
$$

with the spinor $\Psi^{\dagger}=\left(\psi_{\mathbf{r}}^{\dagger}, \psi_{\mathbf{r}}\right)$, and the corresponding single-particle hamiltonian $h\left(\mathbf{r}, \mathbf{r}^{\prime}\right)$ (each being an $8 \times 8$ matrix acting on spin, orbital and Nambu bases). It is easy to show that $h$ satisfies the anti-commutation relation $\left\{\tau_{1} K, h\right\}=0$, where $\tau_{1}$ is the first Pauli matrix acting in the Nambu space and $K$ is the complex conjugation operator. By this antisymmetry, if $h|\Psi\rangle=E|\Psi\rangle$, it is guaranteed that $h \tau_{1} K|\Psi\rangle=-E \tau_{1} K|\Psi\rangle$. Thus $|\Psi\rangle$ and $\tau_{1} K|\Psi\rangle$ form a pair of (orthogonal) states with opposite energies. Because of the doubling of degrees of freedom in the Nambu space, only one of such two states are independent. If $E=0$, the two states become degenerate, and any linear combination of them is also an eigenstate. In a particular combination resulting in a pair of new states $\left|\Psi_{1,2}\right\rangle$, it is possible to enforce $\tau_{1} K\left|\Psi_{1}\right\rangle=\left|\Psi_{1}\right\rangle$ and $\tau_{1} K\left|\Psi_{2}\right\rangle=\left|\Psi_{2}\right\rangle$ (up to trivial phases), in addition to the orthogonality condition $\left\langle\Psi_{1} \mid \Psi_{2}\right\rangle=0$. The quasiparticle field operators associated with these states are self-hermitian, defining the MF modes. They would be stable against perturbations provided they are spatially bounded and not overlapping each other. Such a MF mode was argued to appear in the vortex core in a heterostructure with a layer of s-wave SC on a bulk $\mathrm{TI}^{2}$. The situation we are 
(a)

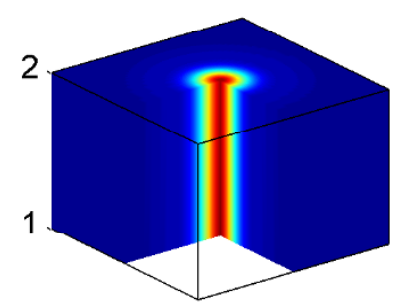

(c)

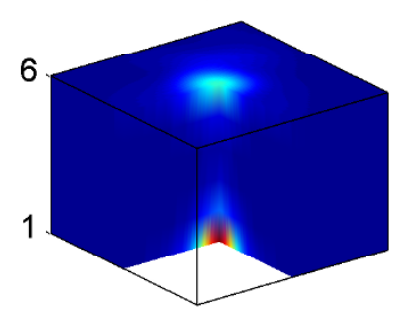

(d)

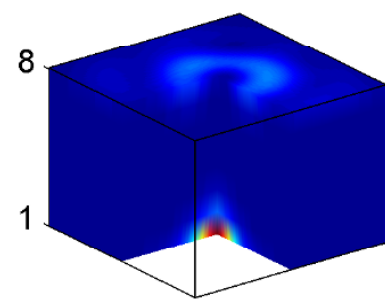

(b)

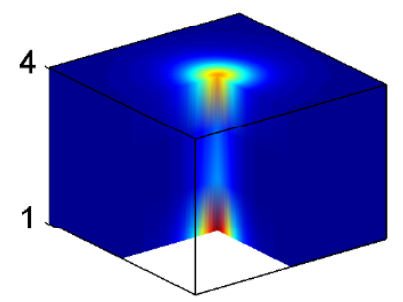

Low
High

Figure $2 \mid$ The wavefunction profile $p_{0}(\mathrm{r})$ for $(\mathrm{a}) n=2$, (b) $n=4$, (c) $n=$ 6 , and (d) $n=8$. In each panel, a corner is cutoff to reveal the interior of the TI.

addressing is upside down in structure: $n$ layers of TI on top of a bulk $s$-wave SC. For sufficiently large $n$, helical states appear on the surfaces of the otherwise isolated TI. By coupling to SC, there should be a MF mode near the interface (the bottom layer of TI) according to Ref. 2. For the same reason another MF mode should appear on the top layer if the proximity induced pairing gap persists over there. For an intermediate $n$, the postulated MF's near the bottom and top layers may couple to each other, lifting the energy degeneracy.

To proceed we investigate numerically the evolution of the lowestlying state $\left|\Psi_{0}\right\rangle$ (with energy $E_{0}$ closest to zero) versus $n$ to gain insights into how MF states would emerge out of $\left|\Psi_{0}\right\rangle$ and $\tau_{1} K\left|\Psi_{0}\right\rangle$. We define the probability density function

$$
p_{0}(\mathbf{r})=\operatorname{Tr}\left|\Psi_{0}(\mathbf{r})\right\rangle\left\langle\Psi_{0}(\mathbf{r})\right|,
$$

to reveal the detailed spatial profile of the emerging MF wavefunction(s) in the heterostructure. Here the trace is taken over spin, orbital and Nambu degrees of freedom. We notice that $p_{0}(\mathbf{r})$ is identical for both $\left|\Psi_{0}\right\rangle$ and $\tau_{1} K\left|\Psi_{0}\right\rangle$, although they are necessarily orthogonal if $E_{0} \neq 0$. In the calculations we fix the parameters $\left(t_{\|}, t_{\perp}, m, \mu\right.$, $\left.\Delta_{0}, \xi_{0}\right)=(1,0.5,0.3,0.23,0.1,10)$ without lost of generality. The smaller hopping $t_{\perp}$ takes qualitative account of the anisotropy in real samples. Fig. 2 shows the resulting $p_{0}(\mathbf{r})$ (color scale) for $n=2,4,6,8$ and $L=150=15 \xi_{0}$. We see that the state is bounded to the vortex line in all cases. It is fully connected across the layers for $n=2$ in Fig. 2(a). In Fig. 2(b), $p_{0}(\mathbf{r})$ is mainly concentrated near the top and bottom layers, implying that this is not the usual Andreev bound state, but rather two MF states slightly reconstructed due to the coupling in the interior of the sample. Indeed, a separate calculation shows that in an isolated $n \geq 4$ sample, gapless helical surface states begin to show up. Such states coupled to the pairing potential would lead to MF states. For larger $n$ in Fig. 2(c) and (d), the function $p_{0}(\mathbf{r})$ becomes more disconnected along $z$ due to weaker coupling between the top and bottom layers, and at the same time more extended on the top layer where the effective pairing gap decreases for increasing $n$. Since an extended MF mode would be fragile, the above results suggest that there is an optimal value of $n$, say $n_{o p t}$, to look for MF mode on the top layer of the present heterostructure. Since the energy $E_{0}$ is nonzero in our finite-sized sample, we check the scaling of $E_{0}$ versus $1 / L$ in Fig. 3. Clearly $E_{0}$ is finite and asymptotically independent of $L$ in the limit of large $L$ for $n=2$, while $E_{0} \rightarrow 0$ with increasing $L$ for $n=6$. The case for $n=4$ appears marginal due to the coupling between top and bottom layers. Thus we expect a genuine MF mode on the top layer for $n \geq n_{\text {opt }}=6$ in the thermodynamic limit of $L$, supporting the experimental setup in search of MF. Of course the above $n_{\text {opt }}$ should only be taken qualitatively, given the difficulty to extract the exact parameters in the experiment.

Before closing this section we remark that since the top and bottom MF's are bound to the vortex line, braiding two vortices leads to braiding of the MF pairs. The statistics during such a braiding is identical to that for conventional fermions. Thus in the present heterostructure it might be difficult to achieve non-Abelian statistics that requires braiding individual MF's.

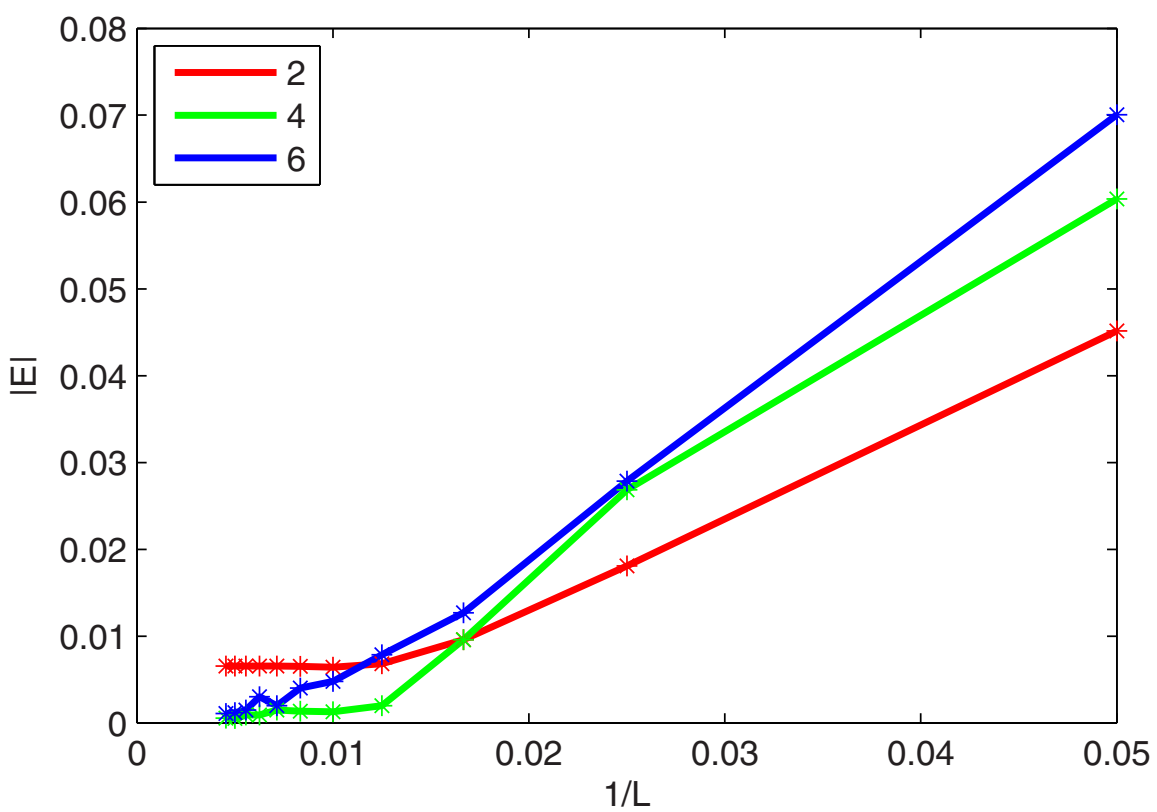

Figure $3 \mid$ Finite size scaling of $E_{0}$ versus $1 / L$ for various $n$. Symbols are the data and lines are guides to the eye. 
Effective 2d models. In the experiment the LDOS is measured by STM. Since the contribution mainly comes from the top layer, it is beneficial to investigate the top layer in more details. To reduce the computation load, we resort to an effective 2D model describing the low-lying quasi-particle states. We first gain understanding by analytical analysis of the a continuum model. We then perform numerical simulations in a lattice model. We also study the robustness of the MF state against impurities, and compare the LDOS to the unexpectedly symmetric zero bias peak observed in the experiment.

Analytical treatment in the continuum limit: In the low energy window, the effective hamiltonian in the Nambu space can be written as,

$$
H=\int d^{2} \mathbf{r} \Psi^{\dagger}\left[(-i \nabla \cdot \sigma-\mu) \tau_{z}-\Delta_{1} \tau_{x}+\Delta_{2} \tau_{y}\right] \Psi .
$$

Here we used a special convention for the annihilation field $\Psi=\left(\psi_{\uparrow}, \psi_{\downarrow},-\psi_{\downarrow}^{\dagger}, \psi_{\uparrow}^{\dagger}\right)^{T}$, and the group velocity $v_{D}$ of the helical states is set to unity. Henceforth we use $\mathbf{r}$ to denote the $2 \mathrm{D}$ position. We performed a spin rotation to write $\nabla \cdot \sigma$ instead of $\partial_{x} \sigma_{y}-\partial_{y} \sigma_{x}$. $\Delta_{1}\left(\Delta_{2}\right)$ is the real (imaginary) part of the $s$-wave pairing potential

$$
\Delta(\mathbf{r})=\Delta_{1}(\mathbf{r})+i \Delta_{2}(\mathbf{r})=\Delta_{0} \tanh (r / \xi) e^{i \phi},
$$

where $\Delta_{0}(r)$ is the pairing scale, $\xi$ the coherence length and $\phi$ the azimuthal angle of $\mathbf{r}$. Finally we did not include the vector potential a since for an isolated vortex in an infinite area and in the London limit the effect of a is negligible as far as the low energy bound states are concerned. In the normal state, the hamiltonian leads to the band dispersion $E_{\mathrm{k}}= \pm k-\mu$, where the sign depends on the chirality $\hat{k} \cdot \sigma$. Because of the spin-momentum locking the two bands are spin nondegenerate. Therefore the intra-band pairing must be odd, and turns out to be $p+i p^{\prime}$-like in the present case. This implies immediately a $\mathrm{MF}$ in the vortex core, just as in a $p+i p^{\prime}$-superconductor ${ }^{18}$. The inter-band pairing is irrelevant for $\Delta_{0} \ll|\mu|$.

For our purpose, we need the wavefunctions of the low lying states. Following Volovik ${ }^{18}$, this is most easily obtained in the quasi-classical approximation. By rotation symmetry the states can by classified by the angular momentum $l$. The energy up to the first order of $l$ is $E=$ $-l \omega_{0}$, with

$$
\omega_{0}=\int d s f_{0}^{2}(s) \frac{\Delta_{0} \tanh (r / \xi)}{k_{F} r} \sim \frac{\Delta_{0}}{k_{F} \xi},
$$

where $k_{F}$ is the Fermi momentum and $f_{0}$ is an envelop function

$$
f_{0}(s) \sim \frac{1}{C} \exp \left[-\int_{0}^{s} \Delta_{0} \tanh \left(r^{\prime} / \xi\right) \cos \phi^{\prime} d s^{\prime}\right],
$$

with a normalization constant $C$ and $s=r \cos \phi$. The corresponding wavefunction is

$$
\Psi_{l}(\mathbf{r}) \sim 2 \pi i^{l} f_{0}(r)\left(\begin{array}{c}
J_{l}\left(k_{F} r\right) e^{i l \phi} \\
i J_{l+1}\left(k_{F} r\right) e^{i(l+1) \phi} \\
J_{l-1}\left(k_{F} r\right) e^{i(l-1) \phi} \\
i J_{1}\left(k_{F} r\right) e^{i l \phi}
\end{array}\right)
$$

where $J_{l}$ is the Bessel function. The energy levels obtained here are essentially similar to the Caroli-de Gennes-Matricon states in usual $s$-wave superconductors ${ }^{19}$, except that the angular momentum $l$ here is an integer rather than a half integer due to the fact that the helical state picks up a negative sign upon a rotation by $2 \pi$.

Of particular interest is the state with $l=0$. The general argument presented in the previous section implies this zero energy state must describe a MF mode. Indeed, the creation field operator associated with this state is, up to the envelop function $f_{0}(r)$,

$$
\Psi^{\dagger} \sim J_{0} \psi_{\uparrow}^{\dagger}-i J_{0} \psi_{\uparrow}+i J_{1} e^{i \phi} \psi_{\downarrow}^{\dagger}+J_{-1} e^{-i \phi} \psi_{\downarrow},
$$

which is self-hermitian up to a trivial global phase. In principle there should be another MF mode on the outer boundary of an open system. But this is an artifact of the $2 \mathrm{D}$ model in view of the $3 \mathrm{D}$ heterostructure (where MF's appear on the top and bottom layers).

The electronic LDOS from the above states is given by

$$
\rho(\omega, \mathbf{r}) \propto \sum_{l}\left[J_{l}^{2}\left(k_{F} r\right)+J_{l-1}^{2}\left(k_{F} r\right)\right] f_{0}(r)^{2} \delta\left(\omega-l \omega_{0}\right) .
$$

Since $J_{l}(0)=0$ for $l \neq 0$, the contribution to the spectral weight at $r=$ 0 comes from $l=0$ and $l=1$ at energy $\omega=0$ and $\omega=\omega_{0}$, respectively. Thus there are two LDOS peaks located asymmetrically in energy. The energy of the side peak is positive for $\mu>0$ here, but would be negative if $\mu<0$ instead. The asymmetry in the LDOS is a direct consequence of the absence of the particle-hole symmetry in the normal state band structure since the Fermi level is away from the Dirac point in our case. Similar asymmetries were actually found even for the bound states in the vortex core of usual $s$-wave superconductors as the Fermi level is close to the band edges, both theoretically and experimentally ${ }^{20,21}$. Of course, as the Fermi level approaches the Dirac point $(\mu \rightarrow 0), k_{F}$ decreases and thus the level spacing $\omega_{0}$ in our case increases. As $k_{F} \rightarrow 0$, the quasi-classical approximation fails but the tendency of $\omega_{0} \propto 1 / k_{F}$ does imply the absence of in-gap bound states other than the unique Majorana zero mode for $k_{F}=0$, as discussed elsewhere ${ }^{15-17}$. But this is clearly not the case in the experiment, where many bound states are observed ${ }^{14}$.

As we step away from the vortex core, the other states with higher $l$ contributes, and from the behavior of Bessel functions, LDOS peaks should appear at the guiding centers of $J_{l, l \pm 1}\left(k_{F} r\right)$ for $\omega= \pm l \omega_{0}$. Such a peak splitting behavior is similar to that in usual superconductors. Rather unexpectedly the experiment reports a symmetric zero-bias peak, and it appears that the central peak does not split until $r$ is a few atoms away from the vortex core ${ }^{14}$. One possible reason for the discrepancy is the working temperature is higher than $\omega_{0}$ that smears out the asymmetry due to the side peak. Another possibility is impurities pinning the vortex may have suppressed the $l=1$ state locally, leaving the $l=0$ Majorana state intact. We shall come back to this point later.

Lattice model: We now consider an effective 2D lattice model for the low-lying states on the top layer of the heterostructure. The effective hamiltonian is,

$$
\begin{aligned}
H_{0}= & \frac{1}{2} \sum_{\mathbf{r}, \mathbf{b}}\left[\psi_{\mathbf{r}}^{\dagger} t_{\mathbf{b}}\left(i \sigma_{x} \delta_{\mathbf{b}, \hat{y}}-i \sigma_{y} \delta_{\mathbf{b}, \hat{x}}-\sigma_{z}\right) \psi_{\mathbf{r}+\mathbf{b}}+\text { h.c. }\right] \\
& +\sum_{\mathbf{r}} \psi_{\mathbf{r}}^{\dagger}\left[2 t \sigma_{z}-\mu\right] \psi_{\mathbf{r}},
\end{aligned}
$$

where $\mathbf{b}=\hat{x}, \hat{y}$. We set $t_{\mathbf{b}}=t=1$ and $\mu=0.23$. Notice that the $\sigma_{z}$ terms are necessary to make sure that there is only one Dirac point in the Brillouine zone. Such terms violate time-reversal symmetry. Nonetheless, this effect is negligible for low lying states close to the Dirac point. In the presence of vortex, we couple the vector potential to electrons by minimal coupling as described in the previous section. We use a large lattice (of linear dimension $L=400$ in this subsection) to reduce finite size effect. The pairing hamiltonian $H_{p}$ is of a form closely similar to that in Eq.8, except that $\mathbf{r}$ is a $2 \mathrm{D}$ vector here. From now on we switch back to the convention $\Psi^{\dagger}=\left(\psi_{\uparrow}^{\dagger}, \psi_{\downarrow}^{\dagger}, \psi_{\uparrow}, \psi_{\downarrow}\right)$ in the Nambu space. The LDOS is given by

$$
\rho(\mathbf{r}, \omega)=\sum_{n} \delta\left(\omega-E_{n}\right) A_{n}(\mathbf{r}),
$$

where 


$$
A_{n}(\mathbf{r})=\operatorname{Tr} \frac{\tau_{0}+\tau_{3}}{2}\left|\Psi_{n}(\mathbf{r})\right\rangle\left\langle\Psi_{n}(\mathbf{r})\right|,
$$

is the level-resolved local spectral weight. In practice, the delta function is smeared by a proper factor. On the other hand, the dimension of the single particle hamiltonian is too large to get all eigenstates. Instead, we use the real-space recursion method ${ }^{22,23}$ to get $\rho(\mathbf{r}, \omega)$ most reliably.

Fig. 4(a) shows $\rho(\mathbf{r}, \omega)$ for $\mathbf{r}$ along a $(1,1)$-cut emanating from the vortex core, and (b) shows a few low energy levels with the height proportional to $A_{n}(\mathbf{r})$. There is clearly a ZBP and a side peak near the vortex core. As discussed previously, the zero energy state must be a MF mode. The energy of the side peak is $E_{1} \sim \Delta_{0} / k_{F} \xi$ where $k_{F} \xi \sim 2.3$ in our case. Moreover, the peaks shift to higher energies as $\mathbf{r}$ is more distant from the core. All of these features are consistent with the previous analytical discussions. In particular, there should be a side peak accompanying the ZBP. It is however not observed in the experiment. Apart from thermal smearing, the discrepancy is likely caused by impurities which we consider in the following.

The impurity hamiltonian can be written as,

$$
\begin{aligned}
H_{i m p} & =\sum_{\mathbf{r}} \Psi_{\mathbf{r}}^{\dagger}\left[\sum_{i=0,1,3} V_{i}(\mathbf{r}) \sigma_{i} \tau_{z}+V_{2}(\mathbf{r}) \sigma_{y} \tau_{0}\right] \Psi_{\mathbf{r}} \\
& \rightarrow \sum_{\mathbf{r}} \Psi_{\mathbf{r}}^{\dagger} V_{i m p}(\mathbf{r}) \Psi_{\mathbf{r}},
\end{aligned}
$$

where $V_{0}(\mathbf{r})$ and $V_{i=1,2,3}(\mathbf{r})$ are the position dependent impurity strengths of scalar and magnetic impurities (of various polarizations), respectively. Here the single-particle part is denoted as $V_{\text {imp }}(\mathbf{r})$ for convenience. We observe that $\left\{V_{\text {imp }}, \tau_{1} K\right\}=0$. For a MF state $|\Psi\rangle$ (satisfying $\tau_{1} K|\Psi\rangle=|\Psi\rangle$ ), the energy correction is, to the first order of $V_{\text {imp }}$,

$$
\begin{aligned}
\Delta E & =\left\langle\Psi\left|V_{i m p}\right| \Psi\right\rangle=\left\langle\Psi\left|V_{i m p} \tau_{1} K\right| \Psi\right\rangle \\
& =-\left\langle\Psi\left|\tau_{1} K V_{i m p}\right| \Psi\right\rangle=-\left\langle\Psi\left|V_{i m p} K \tau_{1}\right| \Psi\right\rangle \\
& =-\left\langle\Psi\left|V_{i m p}\right| \Psi\right\rangle,
\end{aligned}
$$

which must be zero by comparing the first and last lines. Therefore the MF state is immune to weak impurities of any kind. In this sense the MF is both charge and spin neutral. On the other hand, no protection mechanism applies for the energy of other finite-energy bound states against $V_{\text {imp. }}$. Fig. 5 is a similar plot to Fig. 4 but with a local scalar impurity right located at the vortex core. The impurity strength is $V_{0}=15 \Delta_{0}$. (This is a weak impurity since $\Delta_{0}$ is a small energy scale as compared to the bandwidth.) We observe that the previous side peak at energy $E_{1}$ is completely wiped out. The higher energy levels remain intact since their wavefunctions vanish at the vortex core, thus are decoupled to the local impurity to the leading order. The LDOS becomes much more symmetric at the vortex core, (a)

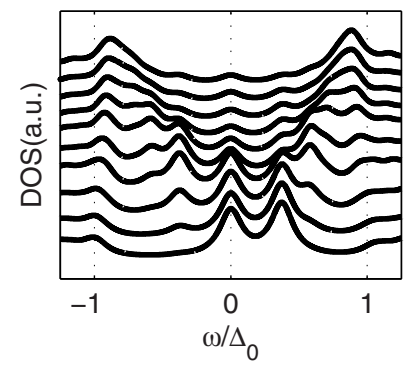

(b)

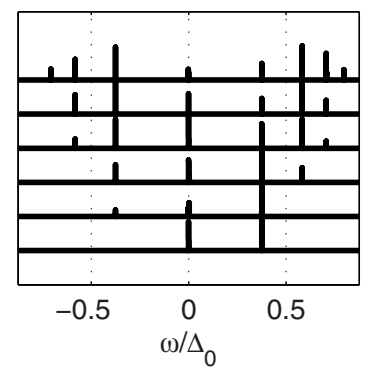

Figure $4 \mid$ (a) LDOS along a $(1,1)$-cut, starting from the vortex core (bottom line). (b) Low energy levels with the height proportional to the respective spectral weight for each point along the cut. (a)

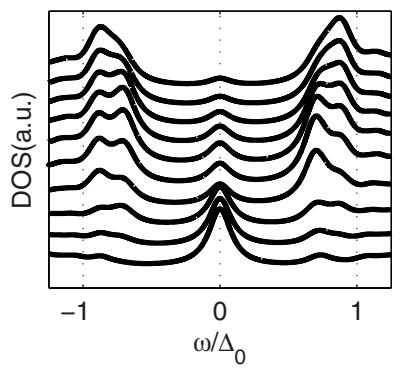

(b)

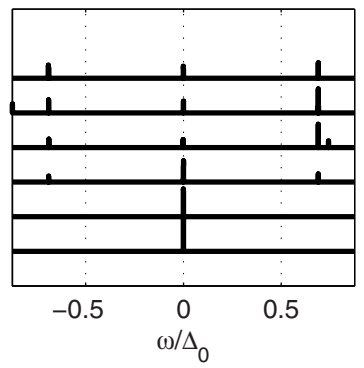

Figure $5 \mid$ The same plot as Fig. 4 except that a local scalar impurity with $V_{0}=15 \Delta_{0}$ is located at the vortex core.

and the peak does not break near the vortex core, in nice agreement with the experiment ${ }^{14}$. We should emphasize, however, that the LDOS in our case is still slightly asymmetric near the gap edges, as shown in Fig. 5b. The asymmetry is an intrinsic feature of the model as the Fermi level is away from the Dirac point, as we discussed analytically, and is enhanced by the relatively large gap scale in the calculation that is necessary to improve the numerical resolution. In the experiment, the gap scale is much smaller than the Fermi energy (the distance from the Fermi level to the Dirac point), so that the energy level spacing of the bound states is smaller. Combined with the finite temperature smearing, the experimental LDOS may appear more symmetric than what we show in Fig. 5. Therefore the difference to the theory is quantitative rather than qualitative.

We also show the corresponding results for a local magnetic impurity in Fig. 6. The impurity reduces the amplitude of the MF mode at the core, but the zero energy level remains robust. On the other hand, additional in-gap states are induced by the impurity. The LDOS is asymmetric in energy due to the side peaks. Experimentally neither the reduced ZBP nor the asymmetry of LDOS is observed, implying the absence of magnetic impurities therein.

Finally we examine the effect of a finite concentration of impurities, an issue also relevant to experiments. Since the impurities too afar from the vortex core are irrelevant for the low-lying bound states, we put impurities within a radius $R=10$ around the core randomly, with ensemble averages

$$
\left\langle V_{i}(\mathbf{r}) V_{j}\left(\mathrm{r}^{\prime}\right)\right\rangle=W_{i}^{2} \theta(R-r) \delta_{\mathbf{r}, \mathbf{r}^{\prime}} \delta_{i j}
$$

where $W_{i}$ is the strength of type $i$. For magnetic impurities we define $W_{m}=\sqrt{\sum_{i=1,2,3} W_{i}^{2}}$ as a measure of the total strength. By monitoring the presence/absence of the zero energy level, we obtain a phase diagram in Fig. 7, showing the critical strength $W_{c}$ of $W_{0}\left(W_{m}\right)$ for scalar (magnetic) impurities, above which the MF disappears, as a function of the impurity concentration $c$. The critical strength is reduced to the order of gap scale for both scalar and magnetic impur- (a)

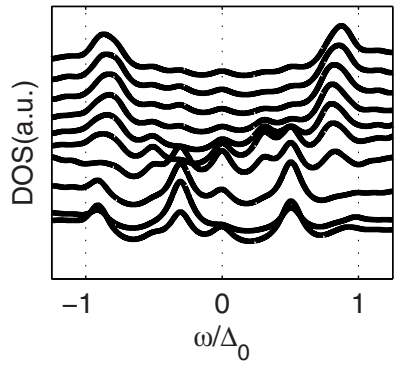

(b)

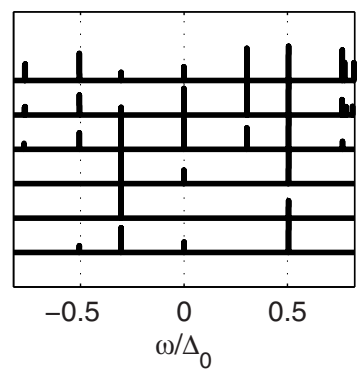

Figure 6 The same plot as Fig. 4 except that a local magnetic impurity with strength $V_{X}=12 \Delta_{0}$ is located at the vortex core. 


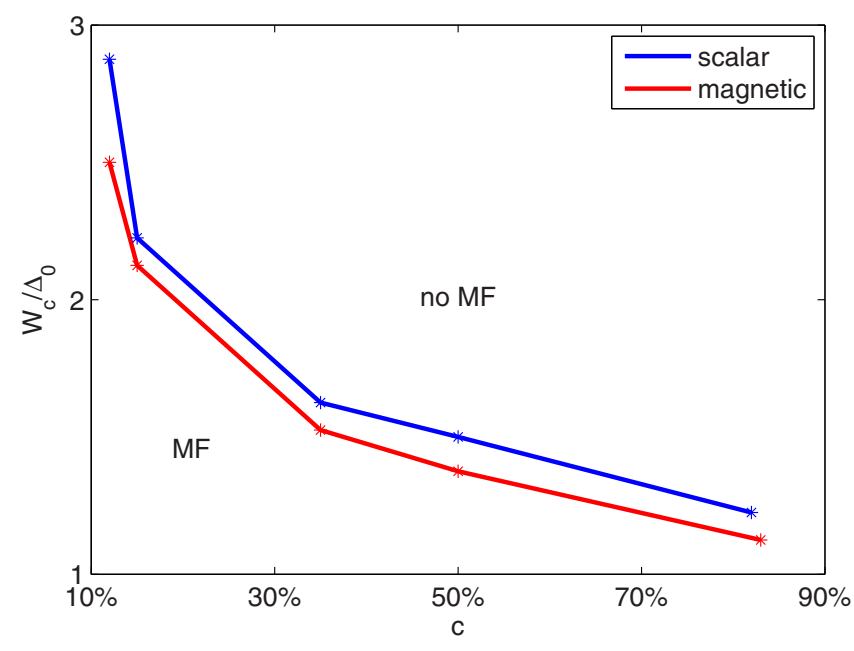

Figure $7 \mid W_{c}$ versus $c$ for scalar (red) and magnetic (blue) impurities.

ities. This is in contrast to the stability in the single impurity case. The result suggests that high sample quality is necessary.

\section{Summary}

In conclusion, we provided strong theoretical support to the experimental result that there exists an optimal number $\left(n_{\text {opt }}\right)$ of TI layers for MF to appear on the top layer of the TI/SC heterostructure. We point out however that the MF modes on the top and bottom TI layers are bound to the vortex line and would cause difficulty to achieve braiding individual MF's. We show analytically and numerically that the LDOS near the vortex core has a ZBP and a side peak. The absence of the side peak in the experiment is nicely reconciled by the effect of a local scalar impurity pinning the vortex core. We also examined the effect of a finite concentration of impurities. While the MF mode is extremely robust to a single local impurity, the critical impurity strength drops drastically to the order of the effective pairing gap at a moderate impurity concentration. This points toward the importance of sample quality. We suggest that the effect of single and many impurities be tested by STM (at sufficiently low temperatures) intentionally to corroborate the MF mode further and to examine its stability, paving the way toward the application of such fascinating objects.

1. Wilczek, F. Majorana returns. Nat. Phys. 5, 614 (2009).

2. Fu, L. \& Kane, C. L. Superconducting proximity effect and Majorana fermions at the surface of a topological insulator. Phys. Rev. Lett. 100, 096407 (2008).

3. Lutchyn, R. M., Sau, J. D. \& Das Sarma, S. Majorana fermions and a topological phase transition in semiconductor-superconductor heterostructures. Phys. Rev. Lett. 105, 077001 (2010).

4. Silaevm, M. A. \& Volovik, G. E. Topological superfluid ${ }^{3} \mathrm{He}-\mathrm{B}$ :fermion zero modes on interfaces and in the vortex core. J. Low Temp. Phys. 161, 460 (2010).

5. Ivanov, D. A. Non-Abelian statistics of half-quantum vortices in p-wave superconductors. Phys. Rev. Lett. 86, 268 (2001).

6. Nayak, C., Simon, S. H., Stern, A., Freedman, M. \& Das Sarma, S. Non-Abelian anyons and topological quantum computation. Rev. Mod. Phys. 80, 1083 (2008).

7. Kitaev, A. Y. Unpaired Majorana fermions in quantum wires. Phys.-Uspekhi 44, $131(2001)$
8. Kwon, H.-J., Sengupta, K. \& Yakovenko, V. M. Fractional ac Josephson effect in pand d-wave superconductors. Eur. Phys. J. B 37, 349 (2004).

9. Law, K. T., Lee, P. A. \& Ng, T. K. Majorana fermion induced resonant Andreev reflection. Phys. Rev. Lett. 103, 237001 (2009).

10. Wimmer, M., Akhmerov, A. R., Dahlhaus, J. P. \& Beenakker, C. W. J. Quantum point contact as a probe of a topological superconductor. New J. Phys. 13, 053016 (2011).

11. Nilsson, J., Akhmerov, A. R. \& Beenakker, C. W. J. Splitting of a Cooper pair by a pair of Majorana bound states. Phys. Rev. Lett. 101, 120403 (2008).

12. Liu, J., Zhang, F. C. \& Law, K. T. Majorana fermion induced nonlocal current correlations in spin-orbit coupled superconducting wires. Phys. Rev. B 88, 064509 (2013).

13. Wang, M. X. et al. The coexistence of superconductivity and topological order in the $\mathrm{Bi}_{2} \mathrm{Se}_{3}$ thin films. Science 336, 52 (2012).

14. Xu, J. P. et al. Majorana mode in vortex core of $B i_{2} \mathrm{Te}_{3} / \mathrm{NbSe}_{2}$ topological insulatorsuperconductor heterostructure. arXiv, 1312.7110 (2013).

15. Chiu, C. K., Gilbert, M. J. \& Hughes, T. L. Vortex lines in topological insulatorsuperconductor heterostructures. Phys. Rev. B 84, 144507 (2011).

16. Tagliacozzo, A., Lucignano, P. \& Tafuri, F. Superconductive proximity in a topological insulator slab and excitations bound to an axial vortex. Phys. Rev. B 86, 045435 (2012).

17. Parente, V., Campagnano, G., Giuliano, D., Tagliacozzo, A. \& Guinea, F. Topological defects in topological insulators and bound states at topological superconductor vortices. Materials 7, 1652 (2014).

18. Volovik, G. E. Fermion zero modes on vortices in chiral superconductors. Pisma Zh. Eksp. Teor. Fiz. 70, 601-606 (1999); JETP Lett. 70, 609 (1999).

19. Caroli, C., de Gennes, P. G. \& Matricon, J. Bound fermion states on a vortex line in a type II superconductor. Phys. Lett. 9, 307 (1964).

20. Nishimori, H. et al. First observation of the fourfold-symmetric and quantum regime vortex core in $\mathrm{YNi}_{2} \mathrm{~B}_{2} \mathrm{C}$ by scanning tunneling microscopy and spectroscopy. J. Phys. Soc. Jpn. 73, 3247 (2004).

21. Hayashi, N., Isoshima, T., Ichioka, M. \& Machida, K. Low-lying quasiparticle excitations around a vortex core in quantum limit. Phys. Rev. Lett. 80, 2921 (1998).

22. Wang, D., Xu, J., Xiang, Y. Y. \& Wang, Q. H. Electronic structure around a vortex core in iron pnictide superconductors. Phys. Rev. B 82, 184519 (2010).

23. Cheng, M. \& Su, W. P. Local density of states and angle-resolved photoemission spectral function of an inhomogeneous d-wave superconductor. Phys. Rev. B 72, 094512 (2005).

\section{Acknowledgments}

We are grateful to $\mathrm{L}$. Fu for helpful discussions on the bound states in a vortex core, and to J.F. Jia for discussions on the experiment. The project was supported by NSFC (under grant No.11023002 and No.11274269), and the Ministry of Science and Technology of China (under grant No.2011CBA00108 and 2011CB922101).

\section{Author contributions}

Z.Z.L. performed the calculations and provided all of the figures. F.C.Z. and Q.H.W initiated the project. Q.H.W. wrote the manuscript. All authors reviewed the manuscript

\section{Additional information}

Competing financial interests: The authors declare no competing financial interests.

How to cite this article: Li, Z.-Z., Zhang, F.-C. \& Wang, Q.-H. Majorana modes in a topological insulator/s-wave superconductor heterostructure. Sci. Rep. 4, 6363; DOI:10.1038/srep06363 (2014)

This work is licensed under a Creative Commons Attribution-NonCommercialNoDerivs 4.0 International License. The images or other third party material in this article are included in the article's Creative Commons license, unless indicated otherwise in the credit line; if the material is not included under the Creative Commons license, users will need to obtain permission from the license holder in order to reproduce the material. To view a copy of this license, visit http:// creativecommons.org/licenses/by-nc-nd/4.0/ 